\title{
CLASIFICACIÓN Y ANÁLISIS DE LOS PROCESOS DE CAMBIO EN LA FORMA URBANA DE LAS ÁREAS METROPOLITANAS ESPAÑOLAS: APLICACIÓN DE MÉTRICAS ESPACIALES
}

Verónica Pascual Rosa

Universidad de Alcalá

veronicapascualrosa@hotmail.com

ORCID iD: https://orcid.org/0000-0002-1103-5909

Francisco Aguilera-Benavente

Universidad de Alcalá

f.aguilera@uah.es

ORCID iD: https://orcid.org/0000-0001-5710-2057

\section{Maria Jesús Salado García}

Universidad de Alcalá

mariaj.salado@uah.es

ORCID iD: https://orcid.org/0000-0002-0314-5390

Recibido: 27/04/2017; Aceptado: 18/03/2019.

Cómo citar este artículo/Citation: Pascual Rosa, V., Aguilera-Benavente, F. y Salado García, M. J. (2019). Clasificación y análisis de los procesos de cambio en la forma urbana de las áreas metropolitanas españolas: aplicación de métricas espaciales. Estudios Geográficos, 80 (286), e006. https://doi.org/10.3989/estgeogr.201926.006

RESUMEN: La delimitación y clasificación de las Áreas Metropolitanas ha sido objeto de múltiples estudios, no siendo el caso español ajeno a dicha realidad. No obstante, no son muchos los estudios que abordan esta clasificación a partir de la caracterización de los patrones espaciales que exhibe la ocupación urbana de dichas áreas. El presente estudio presenta un ejercicio de análisis de las formas de 46 Áreas Metropolitanas en España mediante métricas espaciales para la caracterización de la estructura del paisaje en tres fechas diferentes, a partir de la cartografía Corine Landcover. Derivado de dicho análisis, y mediante un ACP y análisis de conglomerados se identificaron las principales características espaciales que permitían caracterizar las Áreas Metropolitanas y se generó una clasificación de éstas, de acuerdo a su ocupación urbana en las tres fechas. Finalmente, el análisis de los cambios de grupo en la clasificación a lo largo de las diferentes fechas permitió identificar y caracterizar diferentes procesos de expansión urbana, que junto con otros análisis pueden ser útiles en los procesos de planificación metropolitanos.

PALABRAS CLAVE: Áreas Metropolitanas españolas; métricas espaciales; clasificación; procesos de crecimiento urbano.

\section{CLASSIFICATION AND ANALYSIS OF URBAN FORM CHANGE IN SPANISH METROPOLITAN AREAS THROUGH SPATIAL METRICS}

ABSTRACT: Many studies have addressed the delimitation and classification of metropolitan areas (MAs) above the world, some of them focused on the Spanish MAs. However, there is still lack of studies addressing MAs classification through the analysis of the spatial patterns of the urban landcover. This study presents an analysis and classification of the spatial patterns for 46 MAs Spain using spatial metrics, PCA, and cluster analysis in 1990, 2000 and 2006. Finally, changes in groups identified allowed the inference of spatial patterns change processes. The authors believe this analysis may be useful for the metropolitan planning process.

KEY WORDS: Spanish Metropolitan Areas; Spatial metrics; classification; urban growth processes. 


\section{INTRODUCCIÓN}

La urbanización es un fenómeno territorial y socioeconómico universal, que afecta tanto a los países desarrollados como en desarrollo (Deng, Wang, Hong y Qi, 2009; Huang, Lu y Sellers, 2007). Con un crecimiento casi exponencial desde finales del s.XIX (Antrop, 2004), los procesos de crecimiento urbano son y seguirán siendo uno de los temas cruciales del cambio global (Grimm et al, 2008; Deng et al., 2009). La expresión de este fenómeno en la UE se traduce en tasas de población en áreas urbanas en torno al $72 \%$ según la Comisión Europea (CE, 2016) a las que España no permanece ajena. No en vano, España ha sido uno de los países que ha experimentado mayor porcentaje de crecimiento de las áreas artificiales en los últimos años en el ámbito europeo, junto con Irlanda y Portugal, de acuerdo con la Agencia Ambiental Europea (EEA, 2006). Entre otros motivos, esta elevada expansión de las zonas urbanas ha sido impulsada por el crecimiento urbano asociado a la burbuja inmobiliaria (Burriel, 2013), lo que a su vez ha incrementado el interés por el estudio de los procesos de expansión urbana y el análisis de las Áreas Metropolitanas (AM) españolas. A este respecto, algunos de los trabajos más interesantes son los desarrollados por Boix-Domenech (2007), Carreras, Otero y Ruiz, (2009), Feria (2004, 2008, 2013, 2015), Miramontes y Vieira (2016), Roca, Moix y Arellano (2012), Salom y Albertos (2010), o el Atlas Digital de las Áreas Urbanas de España (Ministerio de Fomento, 2016), entre otros, donde se aborda tanto el problema de la delimitación de las AM, así como el de su taxonomía o clasificación. ${ }^{1}$

En lo que respecta a la delimitación de AM, los trabajos citados exploran la definición de los límites de las AM mediante diversos tipos de análisis, basados por ejemplo en el tamaño poblacional y la densidad (Carreras et al, 2009), en la interacción entre municipios (Roca et al., 2012), en la variable desplazamientos residencia-trabajo (Feria, 2008), en la densidad de la red de calles y carreteras (Salom y Albertos, 2010) o en la combinación de varios de estos aspectos. A este respecto, por ejemplo, Miramontes y Vieira (2016) emplean, para la delimitación de las áreas urbanas de España y Portugal, una combinación de criterios como el tamaño poblacional, la densidad de población, la evolución del número de viviendas y la movilidad.

En lo que respecta a la clasificación o taxonomía de las AM españolas, el Atlas Digital de las Áreas Urbanas de España (Ministerio de Fomento, 2016) lleva a cabo una clasificación de dichas áreas atendiendo a un conjunto amplio de criterios relacionados con es- tadísticas de la población, de vivienda, estructura territorial, análisis de dinámicas territoriales, así como de las redes de transporte, dando lugar a una clasificación en dos grandes categorías: grandes y pequeñas áreas urbanas. Por su parte, Feria (2013) realiza una taxonomía para España empleando variables como el tamaño, estructura, dinámica y organización funcional, que dan lugar a 6 categorías de AM (Regiones Metropolitanas, AM Consolidadas, AM Polinucleares, AM Standard, AM Menores y AM Incipientes). Otros trabajos como los de Boix-Domenech (2007), realizan una clasificación de las AM en 4 niveles (A, B, C y D), atendiendo al criterio de población.

No existen, sin embargo, trabajos que aborden la clasificación de dichas AM desde una perspectiva fundamentalmente basada en la estructura de la morfología urbana, más allá del empleo de estadísticas o datos indexados a nivel municipal, como sí que sucede en otros trabajos desarrollados para toda Europa (ver por ejemplo Schwarz, 2010). Dichos análisis de la forma urbana son posibles gracias a la proliferación del uso de los SIG y la disponibilidad de cartografía asociada a las áreas urbanas, así como a la generalización en la literatura del uso de métricas espaciales (Chen, Li, Zheng, Guan y Liu, 2011; Herold, Couclelis y Clarke, 2005), entendidas como "mediciones derivadas del análisis digital de mapas temáticos-categóricos que exhiben heterogeneidad espacial..." (Herold et al., 2005, p. 374). Dichas métricas han demostrado ser una herramienta útil para cuantificar la forma de las teselas urbanas a partir de mapas temáticos, empleando para ello los productos cartográficos (Herold et al., 2005). A este respecto, múltiples estudios caracterizan o comparan patrones de crecimiento urbano de áreas urbanas empleando métricas espaciales (Huang et al., 2007; Pham y Yamaguchi, 2011; Schwarz, 2010, entre otros).

No obstante, el uso de estas herramientas presenta algunas dificultades, dado el numeroso conjunto de métricas disponibles y el elevado grado de correlación que presentan (Cushman, McGarigal y Neel, 2008). A este respecto, aunque la mayoría de los estudios seleccionan como paso previo a su aplicación, un conjunto reducido de métricas, no existe un consenso en lo que respecta a la elección del conjunto que se utiliza en cada caso (Aguilera, Valenzuela y Botequilha-Leitao, 2011).

En este sentido, el objetivo del presente estudio es realizar una clasificación de 46 AM españolas, mediante el análisis de sus características morfológicas a través de métricas espaciales, y valorando, además, 
si dicha clasificación permanece estable a lo largo del tiempo (período 1990-2006) o por el contrario se producen procesos de cambio. Para ello, se empleó la cartografía CORINE LANDCOVER (CLC) para 1990, 2000, 2006, a partir de la cual se calcularon un conjunto de métricas espaciales para todas las AM seleccionadas.

A continuación, mediante un análisis factorial, se seleccionó un conjunto reducido de métricas que permitía explicar la mayor parte de la variabilidad observada. Posteriormente, mediante un análisis de conglomerados, se clasificaron las AM en las tres fechas disponibles (1990, 2000 y 2006). Finalmente, se analizaron aquellas que experimentaron cambios en el agrupamiento al que fueron asignadas en las diferentes fechas, interpretando dichos cambios como procesos de modificación del patrón espacial

El artículo se organiza de la siguiente forma: el epígrafe 2 presenta la delimitación de las AM empleada, los datos de partida, las métricas espaciales, el análisis factorial para la selección de las métricas relevantes, así como la clasificación de las AM mediante análisis de conglomerados. El epígrafe 3 muestra los resultados y, finalmente, la discusión y las conclusiones se presentan en el epígrafe 4 y 5 respectivamente.

\section{MATERIALES Y MÉTODOS}

\subsection{Las AM españolas y los datos de partida}

Para el desarrollo del trabajo se seleccionó un conjunto de AM españolas, así como su delimitación espacial. Ante la carencia de una delimitación y consideración oficial semejante a la existente en otros países (Feria, 2004), se adoptó la delimitación de 46 AM propuesta en Feria (2008) mediante la aplicación de la variable movilidad residencia-trabajo (figura 1 ).

En lo que respecta a los datos de partida, se emplearon los límites municipales, y la cartografía de usos de suelo CLC para 1990, 2000 y 2006. Dicha cartografía fue empleada entre otras razones por la existencia de una serie temporal amplia (que actualmente incluye CLC 2012 y CLC 2018), que permitía realizar un análisis de los cambios a lo largo del tiempo, así como por la

FIGURA 1

AM ESPAÑOLAS A PARTIR DE FERIA (2008)

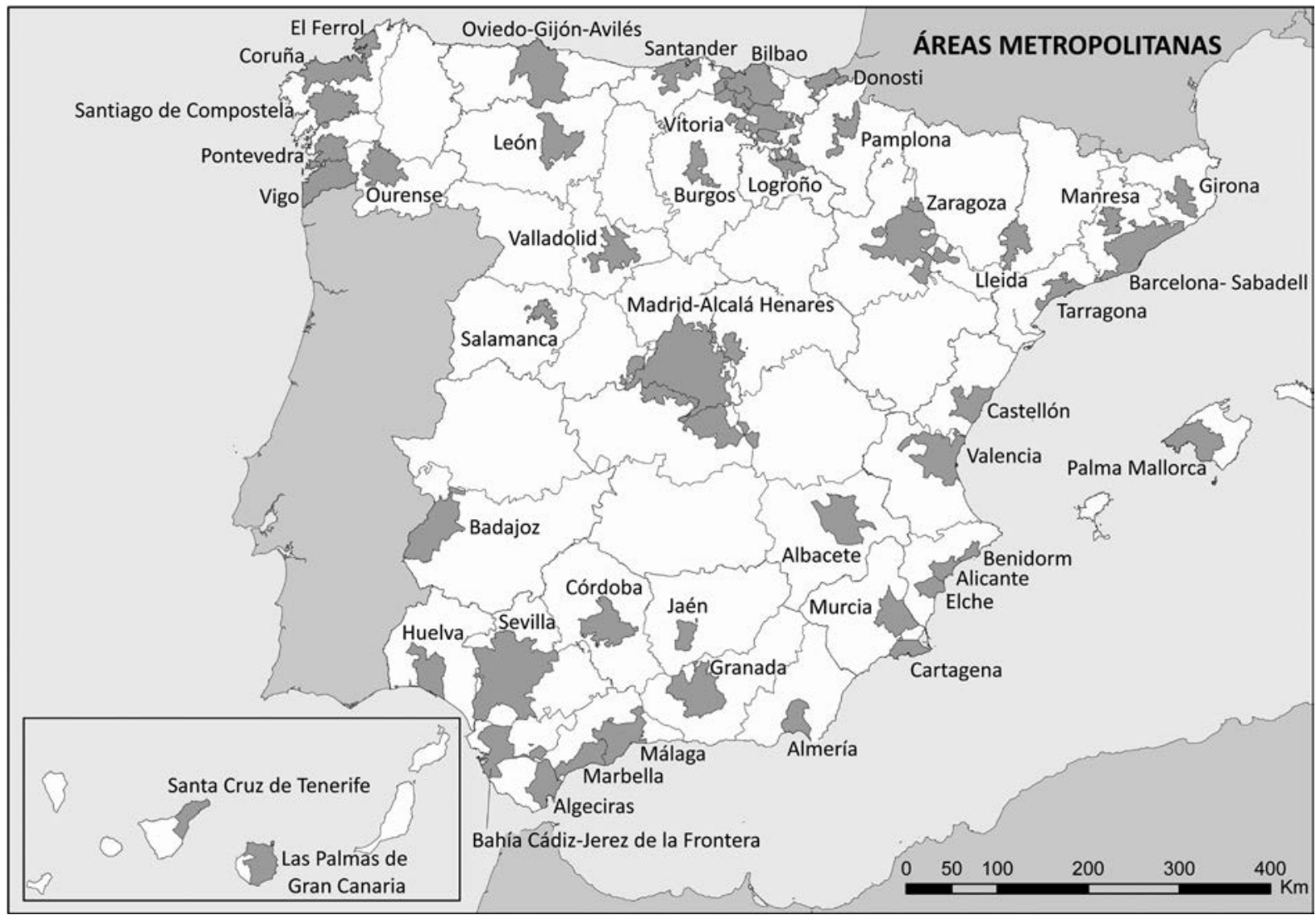


posibilidad de comparar los resultados obtenidos, (a pesar de los posibles errores en dicha cartografía, ver Bach et al, 2006 o Catalá, Bosque y Plata, 2008) con otras áreas metropolitanas europeas.

Para el cálculo de las métricas, fue necesario convertir la cartografía CLC a formato raster, con un tamaño de píxel de 100m. De acuerdo con Pascual, Aguilera y Salado (2014), dicho tamaño de píxel es el más adecuado para el cálculo de las métricas a partir de CLC. De esta forma, las diferentes teselas o fragmentos urbanos extraídos de la cartografía CLC, y que posteriormente se analizaron mediante el cálculo de las métricas espaciales, quedaron representados por grupos de píxeles adyacentes de 100×100m.

Por otra parte, las categorías de CLC correspondientes a usos urbanos se agruparon previamente en una única categoría, sobre la que se realizarán todos los análisis de características espaciales. De este modo, para cada área metropolitana se dispuso de una cartografía de ocupación urbana del suelo en las tres fechas en cuestión.

\subsection{Metodología}

La metodología se articula en las siguientes etapas: i) cálculo de un conjunto amplio de métricas espaciales para las 46 AM españolas, para las que se dispuso de la cartografía CLC (1990, 2000 y 2006), que permitieron cuantificar múltiples características espaciales de las mismas; ii) análisis factorial de los resultados obtenidos, para identificar un conjunto reducido de dimensiones o factores espaciales ${ }^{2}$ que agrupen diversas métricas espaciales altamente correlacionadas; iii) clasificación de las AM mediante análisis de conglomerados, empleando para ello las dimensiones espaciales anteriormente identificadas; iv) análisis de los cambios entre categorías de las $A M$, que se interpretaron como procesos de cambio espacial. Cada una de dichas etapas se presenta de manera detallada en los epígrafes subsiguientes. El software empleado para la realización de los análisis realizados fue ArcGIS 10.0 para el procesado de la cartografía de ocupación urbana elaborada a partir de CLC; Fragstats 4.0 (McGarigal, Cushman y Ene, 2012) para el cálculo de las métricas espaciales, así como Statgraphics, para el cálculo del análisis factorial y el análisis de conglomerados posterior.

\subsubsection{Cálculo de las métricas espaciales}

Las métricas espaciales permiten cuantificar diferentes aspectos espaciales de mapas categóricos, como, por ejemplo, el número total de teselas ur- banas, su tamaño medio, etc. (Botequilha-Leitao y Ahern, 2002). No obstante, la primera dificultad que emerge al emplearlas es el elevado número de ellas existente. Por ello, la selección del conjunto de métricas se convierte en una cuestión central (Cushman et al 2008). A este respecto, en múltiples de los trabajos existentes en la literatura se recurre a emplear métricas basándose en otros trabajos anteriores, o simplemente mediante el argumento de ser las más empleadas en la literatura. En este estudio se ha partido inicialmente de un conjunto de 15 métricas espaciales, de interpretación sencilla, que representan varias de las dimensiones espaciales del paisaje identificadas por Cushman et al (2008), y cuya presencia es frecuente en la literatura científica (tabla 1), para posteriormente realizar una selección de estas.

\subsubsection{El análisis factorial}

Calculado el conjunto inicial de métricas, se realizó un análisis factorial que redujese su número mediante la identificación de dimensiones o factores espaciales que agrupen a varias de ellas (Schwarz, 2010; Aguilera-Benavente y Botequilha-Leitao, 2012; AguileraBenavente et al, 2014). Estas dimensiones fueron las características espaciales que mejor explican la variabilidad espacial de las AM españolas. El método seleccionado para la identificación de dichas dimensiones fue el método de componentes principales, garantizándose así que los factores resultado del mismo no estuvieran correlacionados entre sí.

\subsubsection{La clasificación y caracterización de los tipos de AM}

Los resultados de los factores seleccionados en el análisis factorial para cada una de las AM y para las tres fechas fueron a continuación estandarizados a valores entre 0 y 1 . Dichos valores se sometieron a un análisis de conglomerados para agrupar aquellas AM con características espaciales similares, generándose una única clasificación para las tres fechas. Para ello se decidió utilizar el método jerárquico de Ward (Aguilera-Benavente et al, 2014) aplicado con la distancia euclidiana al cuadrado como la medida de distancia. Igualmente, se experimentó con diferentes números de grupos, optando finalmente por aquel número de agrupamientos cuyos resultados fueron mejor interpretables. De esta forma, el resultado obtenido será la inclusión de cada una de las AM españolas en una categoría en cada una de las tres fechas disponibles. Cada una de las categorías identificadas, fue además de descrita de acuerdo con sus características espaciales. 
TABLA 1

DESCRIPCIÓN DE LAS MÉTRICAS SELECCIONADAS

\begin{tabular}{|c|c|c|c|}
\hline Métrica & Acrónimo & Ecuación & Descripción \\
\hline Área total de la categoría & $\mathrm{CA}$ & $C A=\sum_{j=1}^{n} a_{i j}\left(\frac{1}{10.000}\right)$ & $\begin{array}{l}\text { Área total que ocupa una categoría (ha). Suma del } \\
\text { área de todas las teselas }\left(a_{i j}\right) \text { de una misma } \\
\text { categoría }\end{array}$ \\
\hline Porcentaje de paisaje & PLAND & $P L A N D=\frac{\sum_{j=1}^{a} a_{i j}}{A}(100)$ & $\begin{array}{l}\text { Proporción de paisaje que ocupa el área de una } \\
\text { categoría }(\%) \text {. Suma del área de cada tesela }\left(\mathrm{a}_{\mathrm{ij}}\right) \\
\text { dividida por el área total }(\mathrm{A}) \text { del paisaje }\end{array}$ \\
\hline Número de teselas & NP & $N P=n_{i}$ & Numero de teselas de una misma clase \\
\hline Densidad de teselas & PD & $P D=\frac{n_{i}}{A}(10.000)(100)$ & $\begin{array}{l}\text { Densidad de teselas de una misma clase. } \\
\text { Cantidad de teselas de una clase }\left(\mathrm{n}_{\mathrm{i}}\right) \text { por cada } 100 \\
\text { hectáreas de paisaje }\end{array}$ \\
\hline Índice de la tesela más grande & LPI & $L P I=\frac{{ }_{j=1}^{n} \max \left(a_{i j}\right)}{A}(100)$ & $\begin{array}{l}\text { Corresponde al porcentaje de área que ocupa la } \\
\text { tesela más grande de una clase dentro del paisaje }\end{array}$ \\
\hline Longitud de borde & TE & $T E=\sum_{k=1}^{m} e_{i k}$ & $\begin{array}{l}\text { Es la suma total de las longitudes de todos los } \\
\text { bordes de las teselas de una clase }(\mathrm{m})\end{array}$ \\
\hline Densidad de bordes & ED & $E D=\frac{\sum_{k=1}^{m} e_{i k}}{A}(10.000)$ & $\begin{array}{l}\text { Es la suma total de todas las longitudes de borde de } \\
\text { las teselas de una clase, dividido entre el área total } \\
\text { del paisaje }(\mathrm{m} / \mathrm{ha})\end{array}$ \\
\hline Área media de las teselas & AREA_MN & \multirow{2}{*}{$A R E A=a_{i j}\left(\frac{1}{10.000}\right)$} & Área media de las teselas que componen una clase \\
\hline $\begin{array}{l}\text { Área media ponderada por el } \\
\text { área de las teselas }\end{array}$ & AREA_AM & & $\begin{array}{l}\text { Área media ponderada por el tamaño de las teselas } \\
\text { que componen una clase }\end{array}$ \\
\hline Índice de forma medio & SHAPE_MN & \multirow{2}{*}{$S H A P E=\sum_{j=1}^{n} \frac{0,25 P_{i j}}{\sqrt{a_{i j}}}$} & \multirow{2}{*}{$\begin{array}{l}\text { Es el valor medio de la relación área-perímetro } \\
\text { ajustada a un cuadrado }\end{array}$} \\
\hline $\begin{array}{l}\text { Índice de forma medio } \\
\text { ponderado por el área }\end{array}$ & SHAPE_AM & & \\
\hline $\begin{array}{l}\text { Índice de dimensión fractal } \\
\text { medio }\end{array}$ & FRAC_MN & \multirow{2}{*}{$F R A C=\frac{2 \ln \left(0,25 p_{i j}\right)}{\ln a_{i j}}$} & \multirow[t]{2}{*}{$\begin{array}{l}\text { Es } 2 \text { veces el logaritmo del perímetro de la tesela } \\
\text { dividido por el logaritmo de su área d }\end{array}$} \\
\hline Índice de & FRAC_AM & & \\
\hline $\begin{array}{l}\text { Distancia media a la tesela más } \\
\text { próxima }\end{array}$ & ENN_MN & \multirow{2}{*}{$E N N=h_{i j}$} & \multirow{2}{*}{$\begin{array}{l}\text { Es la distancia a la tesela más cercana de la misma } \\
\text { clase }\end{array}$} \\
\hline $\begin{array}{l}\text { Distancia media a la tesela más } \\
\text { próxima ponderada por el área }\end{array}$ & ENN_AM & & \\
\hline \multirow{2}{*}{$\begin{array}{l}\text { Los acrónimos MN y AM } \\
\text { indican valores medios y } \\
\text { valores medios ponderados } \\
\text { por el área. No debe } \\
\text { confundirse AM empleado aquí } \\
\text { con el acrónimo de Área } \\
\text { Metropolitana }\end{array}$} & $\mathrm{MN}$ & $M N=\frac{\sum_{j=1}^{n} x_{i j}}{n_{i}}$ & Valor medio \\
\hline & AM & $A M=\sum_{j=1}^{n}\left[x_{i j}\left(\frac{a_{i j}}{\sum_{j=1}^{n} a_{i j}}\right)\right]$ & $\begin{array}{l}\text { Valor medio ponderado por el tamaño de las } \\
\text { teselas }\end{array}$ \\
\hline
\end{tabular}




\subsubsection{Los procesos de transformación}

Finalmente, se analizaron los cambios de categorías que experimentaron las diferentes $\mathrm{AM}$ a lo largo de las tres fechas. En este sentido, la existencia de un cambio de categoría para un AM que es clasificada en una categoría en una fecha, y en otra en la fecha siguiente, podría interpretarse como un proceso de transformación que, debido al crecimiento y desarrollo del AM, ve modificadas sus características espaciales lo suficiente como para ser incluida en un grupo diferente. De esta forma, se podrían interpretar los cambios de categoría como procesos de cambio (ver por ejemplo Aguilera-Benavente et al, 2014) que modifican de manera sustancial la forma urbana del AM y que por tanto pueden caracterizarse espacialmente.

De esta forma, el análisis de todos los cambios de categorías experimentados permitió inferir y caracterizar los procesos de cambio experimentados en las AM españolas (identificados a la escala de CLC), y caracterizados mediante las dimensiones espaciales previamente identificadas.

\section{RESULTADOS}

3.1. Las métricas calculadas. El análisis factorial y la descripción de las dimensiones espaciales relevantes

Como primer resultado del análisis se obtuvieron las métricas para cada AM en las tres fechas señaladas. A continuación, del análisis factorial de las 15 métricas obtenidas para las tres fechas, se extrajeron cinco factores o dimensiones espaciales, los cuales permitieron explicar aproximadamente el 93\% de la varianza total del conjunto de datos. A este respecto, y al obtenerse una medición Kaiser-Meyer-Olkin para muestreo idóneo mayor a 0.60 (concretamente 0.66), se pudo considerar que el análisis era viable para su factorización. Además, para facilitar la interpretación de los factores se realizó una rotación ortogonal mediante el método varimax. En la tabla 2 queda reflejado el porcentaje de varianza que explica cada factor, así como la carga de cada una de las métricas. Aquellas cargas cercanas a la unidad se interpretan como la correlación entre el factor y la métrica en cuestión, pudiendo realizarse una interpretación de las dimensiones espaciales obtenidas en el análisis factorial.

TABLA 2

FACTORES EXTRAÍDOS Y VALORES DE VARIANZA

\begin{tabular}{|c|c|c|c|c|c|c|}
\hline & Factor 1 & Factor2 & Factor 3 & Factor 4 & Factor 5 & \\
\hline \% de Varianza & 44,836 & 20,198 & 14,849 & 6,722 & 6,222 & Comunalidad \\
\hline CA & 0,972846 & 0,150863 & $-0,0193612$ & $-0,0474653$ & 0,116498 & 0,985388 \\
\hline PLAND & 0,324154 & 0,850714 & 0,0969139 & $-0,233226$ & 0,179705 & 0,924871 \\
\hline NP & 0,950094 & 0,224916 & 0,00638432 & $-0,0974383$ & $-0,0798166$ & 0,969172 \\
\hline PD & 0,115165 & 0,895023 & 0,0642376 & $-0,26458$ & $-0,25241$ & 0,952169 \\
\hline LPI & 0,175887 & 0,757842 & 0,188962 & $-0,15534$ & 0,513952 & 0,929245 \\
\hline TE & 0,963877 & 0,214381 & 0,0652362 & $-0,0765695$ & 0,027426 & 0,985889 \\
\hline ED & 0,199829 & 0,90802 & 0,230824 & $-0,25634$ & $-0,0340848$ & 0,984584 \\
\hline AREA_MN & 0,147607 & $-0,0507376$ & 0,239172 & $-0,0202815$ & 0,884735 & 0,864733 \\
\hline AREA_AM & 0,876741 & 0,0651366 & 0,052336 & $-0,0414928$ & 0,361574 & 0,908114 \\
\hline SHAPE_MN & $-0,0765904$ & 0,0739933 & 0,930487 & $-0,108644$ & 0,217756 & 0,936369 \\
\hline SHAPE_AM & 0,289377 & 0,300357 & 0,617186 & $-0,182519$ & 0,43746 & 0,779557 \\
\hline FRAC_MN & $-0,033287$ & 0,0485715 & 0,949431 & $-0,0485807$ & $-0,0931565$ & 0,915925 \\
\hline FRAC_AM & 0,138196 & 0,297945 & 0,767296 & $-0,248039$ & 0,331994 & 0,868355 \\
\hline ENN_MN & $-0,0871286$ & $-0,350668$ & $-0,129458$ & 0,897937 & 0,0509719 & 0,956208 \\
\hline ENN_AM & $-0,100424$ & $-0,296614$ & $-0,220497$ & 0,890688 & $-0,153531$ & 0,963582 \\
\hline
\end{tabular}


La dimensión 1 explica el mayor porcentaje de la varianza total (casi $45 \%$ ). Las mayores cargas recaen sobre las métricas del total del área urbana (CA), número de teselas (NP), longitud total de bordes (TE) y el área media de las teselas urbanas ponderadas por su tamaño (AREA_AM). Cuanto mayor es el valor de esta dimensión, mayor es la superficie urbana total (CA), mayor el número de teselas urbanas (NP), mayor la longitud de bordes (TE) y mayor el tamaño medio ponderado por el área (AREA_AM). Por tanto, podría interpretarse como el tamaño del área metropolitana (figura 2a), puesto que aquellas AM de gran tamaño presentan además una alta superficie urbana (CA), elevado número de teselas (NP) y por tanto elevada longitud de bordes (TE), y además presentan una tesela de gran tamaño constituida por la ciudad principal del área metropolitana, que les confiere un elevado valor del AREA_AM.

En la dimensión 2, las métricas con mayores cargas fueron: la densidad de bordes (ED) y la densidad de teselas (PD), ambas con cargas del 0,9. Este factor o dimensión, que explica el $20 \%$ de la varianza total, se interpreta como la fragmentación de los usos urbanos (figura 2b) en el AM, es decir mide el grado en que los usos urbanos se encuentran divididos en pocas o en muchas teselas urbanas. Aquellas AM con valores altos en esta dimensión estarán compuestas por un elevado número de teselas en relación al tamaño de su hinterland (elevado PD), que darán lugar a una alta densidad de bordes (ED) marcados por el contacto de estas teselas urbanas con los usos no urbanos a su alrededor. Por el contrario, valores bajos en este factor mostrarán AM formadas por pocas teselas o con poca densidad de teselas si su hinterland es muy amplio.

La tercera dimensión espacial, con una varianza explicada de aproximadamente el $15 \%$, se encuentra vinculada a las métricas de forma (SHAPE_MN y SHAPE_AM) y el índice de dimensión fractal (FRAC_MN y FRAC_AM). Por tanto, este factor se puede interpretar como la complejidad de la forma de las teselas urbanas (figura 2c). En este sentido, valores altos de esta dimensión se interpretan como formas más elongadas y complejas en las teselas urbanas, como por ejemplo presentan aquellas AM que se desarrollan a lo largo de la línea de costa o fuertemente condicionadas por la topografía (figura 2c). Valores bajos por su parte representarán teselas con formas similares a las del círculo, que generalmente tenderán también a ser aquellas de menor tamaño.

Las dimensiones 4 y 5 , explican cada una en torno a un $6 \%$ de la varianza total, luego son mucho menos relevantes. Concretamente, la dimensión 4 (figura 2d) se encuentra relacionada con las métricas que miden el aislamiento de las teselas, como la distancia media al vecino más próximo (ENN_MN y ENN_AM) por lo que se podría intepretar como el grado de aislamiento de las teselas que forman el AM. Así, valores altos se interpretarían como teselas urbanas muy separadas unas de otras. Finalmente, en la quinta dimensión la métrica con mayor carga fue el área media de las teselas urbanas (AREA_MN), de tal forma que valores elevados muestran AM donde los fragmentos o teselas urbanas presentan un elevado tamaño medio (figura 2e).

FIGURA 2

DIMENSIONES ESPACIALES PARA LA CARACTERIZACIÓN DE LAS AM

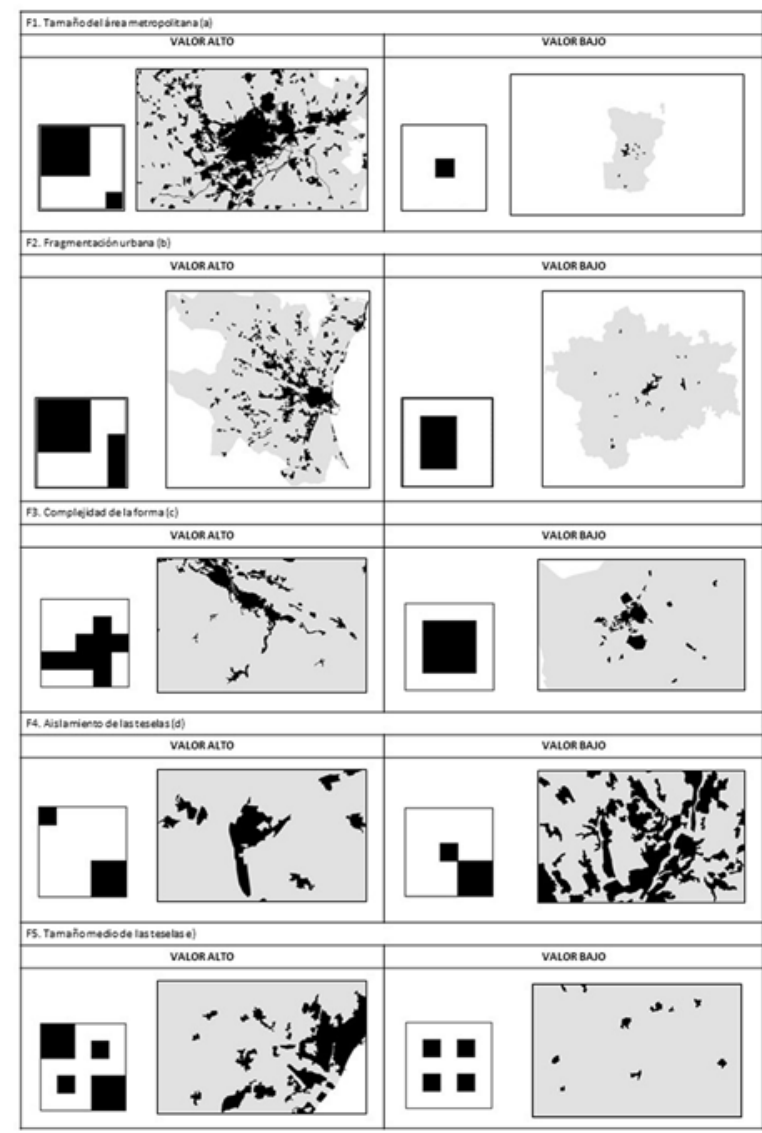

\subsection{La clasificación y caracterización de las AM en el año 2006}

Los resultados del análisis de conglomerados de las 5 dimensiones espaciales para el año 2006 permitieron clasificar las 46 AM en cinco grupos, tras realizar diversas pruebas desde 8 a 4 grupos. La figura 3 muestra el mapa con la clasificación realizada empleando la cartografía CLC para el año 2006, pudiendo observarse en 
este último el grado de proximidad o similitud de unas AM frente a otras. La tabla 3 por su parte resume las características de cada uno de los grupos, los valores obtenidos en cada una de las 5 dimensiones espaciales y algunos ejemplos de AM incluidas en cada categoría.

El primero de los grupos identificados se corresponde con AM de gran tamaño, que adquieren valores altos en todos los factores (tamaño, fragmentación, complejidad de la forma de las teselas y tamaño medio), especialmente del factor de tamaño, y a excepción del factor de aislamiento (tabla 3), que presenta valores bajos dada la proximidad que presentan sus diferentes teselas urbanas. Este grupo está compuesto por AM que tienen una extensión elevada, presentan un número elevado de teselas urbanas, algunas de dichas teselas de gran tamaño, que además presentan formas complejas y que se sitúan unas muy cerca unas de otras (ver figura 4a). Constituyen un paisaje predominantemente urbano con alta fragmentación de sus teselas y muchos espacios intersticiales que no están ocupados por usos urbanos. A este grupo pertenecen las AM de Barcelona y Madrid (figura 4a).
El segundo grupo se ha denominado el de las AM dominadas por la presencia de formas elongadas. Se caracterizan fundamentalmente por los valores más altos en el factor de complejidad de las formas, así como por valores muy bajos para el factor de aislamiento. La mayor parte se corresponden con AM litorales, en las que la topografía condiciona el desarrollo urbano, produciéndose los crecimientos a lo largo de la línea de costa o a lo largo de estrechos valles, dando lugar a las citadas formas alargadas y sinuosas y con teselas poco separadas unas de otras. Un ejemplo de este tipo sería el área metropolitana de Santander (figura 4b), Vigo, Oviedo-Gijón-Avilés, San Sebastián, Málaga, Tenerife, Alicante o Benidorm.

Las AM de formas intermedias se caracterizan por presentar valores medios en todos los factores o dimensiones, sin que parezcan estar caracterizadas por ninguno de los factores en concreto. Las AM de este grupo, en general, no son muy extensas, aunque aparecen excepciones como son las AM de Valencia o Bilbao, y por lo general presentan una fragmentación intermedia Algunos otros ejemplos son Las Palmas de Gran Canaria, A Coruña, Tarragona o Cartagena (figura 4c).

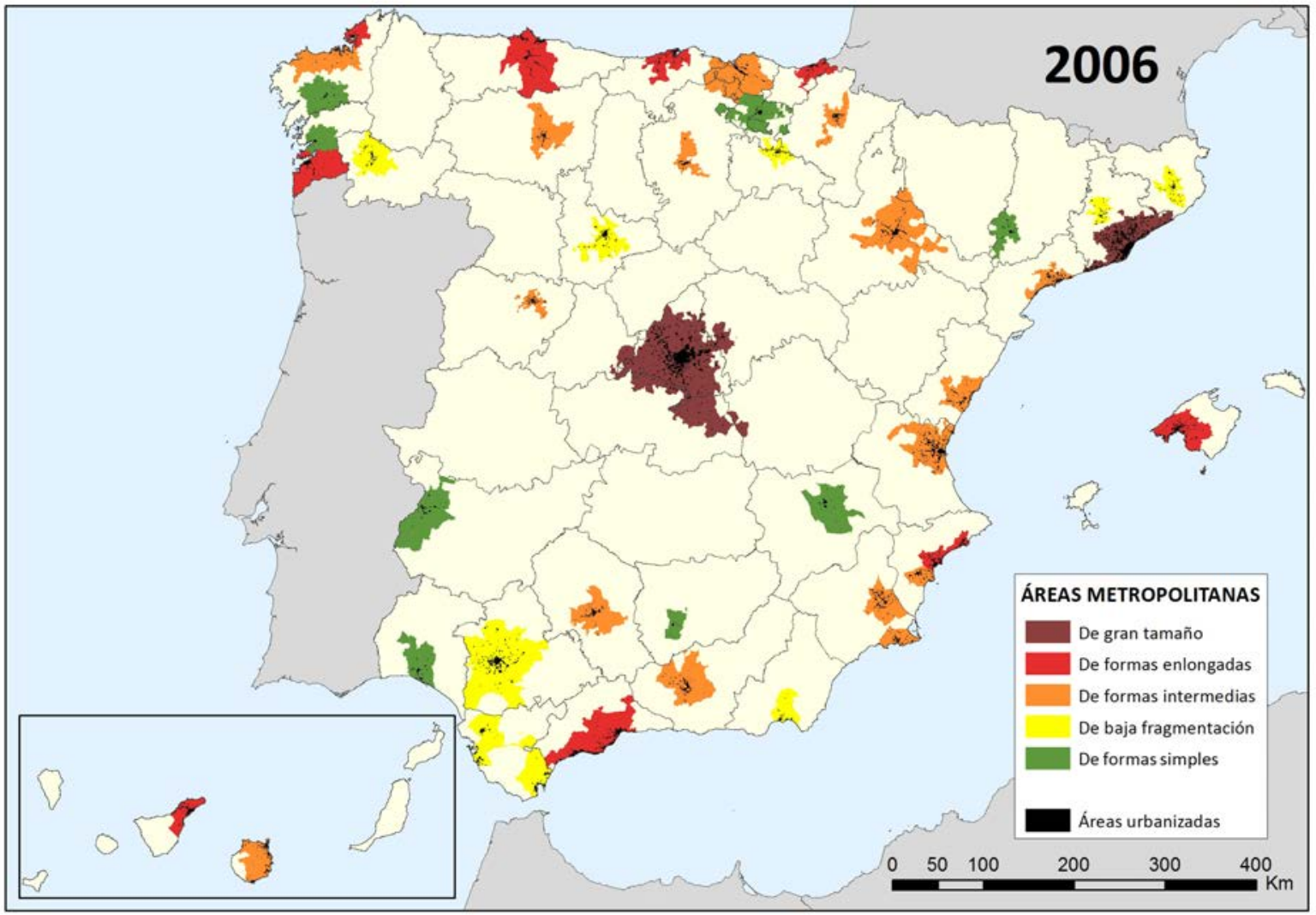


TABLA 3

CLASIFICACIÓN DE LAS AM ESPAÑOLAS

\begin{tabular}{|c|c|c|c|c|c|c|c|c|}
\hline \multirow[b]{2}{*}{ TIPOS DE AM } & \multirow[b]{2}{*}{$\frac{\text { 을 }}{\frac{E}{\omega}}$} & \multirow[b]{2}{*}{ 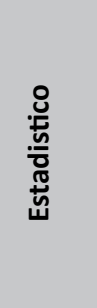 } & \multicolumn{5}{|c|}{ FACTORES } & \multirow[t]{2}{*}{ CARACTERIZACIÓN } \\
\hline & & & 兄 & 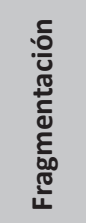 & 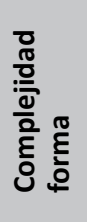 & 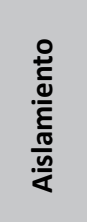 & 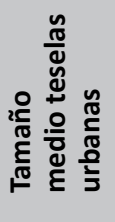 & \\
\hline \multirow{2}{*}{$\begin{array}{l}\text { AM de gran } \\
\text { tamaño }\end{array}$} & \multirow{2}{*}{$\begin{array}{l}\text { Barcelona, } \\
\text { Madrid }\end{array}$} & Media & 15,75 & 9,56 & 4,44 & $-4,18$ & 6,07 & \multirow{2}{*}{$\begin{array}{l}\text { Grupo de AM dominadas } \\
\text { por el elevado valor de la } \\
\text { dimensión del tamaño. Se } \\
\text { trata de grandes AM) }\end{array}$} \\
\hline & & DS & 7,38 & 4,44 & 4,11 & 1,64 & 3,88 & \\
\hline \multirow{2}{*}{$\begin{array}{l}\text { AM dominadas } \\
\text { por la presencia } \\
\text { de formas } \\
\text { elongadas y } \\
\text { sinuosas }\end{array}$} & \multirow{2}{*}{$\begin{array}{l}\text { Palma de } \\
\text { Mallorca; } \\
\text { Santander }\end{array}$} & Media & 0,97 & 3,47 & 4,60 & $-2,44$ & 1,99 & \multirow{2}{*}{$\begin{array}{l}\text { Grupo de AM con el valor } \\
\text { más alto de la dimensión de } \\
\text { complejidad, generalmente } \\
\text { condicionadas por la } \\
\text { topografía }\end{array}$} \\
\hline & & DS & 1,77 & 3,62 & 1,43 & 1,45 & 1,24 & \\
\hline \multirow{2}{*}{$\begin{array}{l}\text { AM de formas } \\
\text { intermedias }\end{array}$} & \multirow{2}{*}{$\begin{array}{l}\text { Granada, } \\
\text { Valencia }\end{array}$} & Media & 0,64 & 1,17 & 0,60 & $-1,05$ & $-0,11$ & \multirow{2}{*}{$\begin{array}{l}\text { Grupo de AM } \\
\text { caracterizadas por valores } \\
\text { intermedios de todos los } \\
\text { factores o dimensiones }\end{array}$} \\
\hline & & DS & 2,46 & 2,43 & 1,22 & 1,08 & 0,85 & \\
\hline \multirow{2}{*}{$\begin{array}{l}\text { AM de baja } \\
\text { fragmentación }\end{array}$} & \multirow{2}{*}{$\begin{array}{l}\text { Sevilla, } \\
\text { Valladolid, } \\
\text { Logroño }\end{array}$} & Media & $-1,35$ & $-1,67$ & $-1,91$ & 0,62 & $-0,90$ & \multirow{2}{*}{$\begin{array}{l}\text { AM dominadas por } \\
\text { la dimensión } 2 \text { de } \\
\text { fragmentación, para el que } \\
\text { presentan valores bajos. }\end{array}$} \\
\hline & & DS & 1,27 & 1,06 & 1,33 & 0,60 & 0,88 & \\
\hline \multirow{2}{*}{$\begin{array}{l}\text { AM de formas } \\
\text { simples }\end{array}$} & \multirow{2}{*}{$\begin{array}{l}\text { Albacete, } \\
\text { Huelva }\end{array}$} & Media & $-3,19$ & $-4,62$ & $-3,71$ & 3,65 & $-1,89$ & \multirow{2}{*}{$\begin{array}{l}\text { AM caracterizadas } \\
\text { por el escaso valor de } \\
\text { todas las dimensiones, } \\
\text { especialmente, dado su } \\
\text { reducido tamaño. }\end{array}$} \\
\hline & & DS & 0,55 & 1,35 & 1,87 & 2,36 & 0,74 & \\
\hline
\end{tabular}

Las AM de escasa fragmentación son un grupo de AM heterogéneo, pero que comparten valores bajos del factor de fragmentación. Esto sucede en dos casos fundamentales: i) cuando las AM tienen un estado de desarrollo poco evolucionado, sin que los fragmentos urbanos alrededor del núcleo principal sean muy numerosos AM de Algeciras, Girona o Logroño, figura 4d); ii) en el caso de AM de mayor grado de desarrollo (incluso AM de gran entidad como es el caso de SeviIla), en las que su delimitación es muy extensa y/o el núcleo principal aglutina gran parte de la ocupación urbana, lo que provoca que la densidad de teselas urbanas sea baja (dada la gran superficie de delimi- tación). Ejemplos de AM que se encuentran en esta situación serían Sevilla o Zaragoza.

Finalmente, las AM de formas simples son las que menores valores presentan en todos los factores, a excepción de los valores del factor del aislamiento, que son los más altos al estar los escasos fragmentos urbanos muy separados unos de otros. Se trata, por tanto, de AM con fragmentos o teselas urbanas escasas, de reducidas dimensiones y alejadas entre sí. Al ser teselas de pequeño tamaño presentan contornos simples y poco elongados. Ejemplo de este tipo serían las AM de Jaén, Vitoria, Huelva, Santiago, Badajoz, Lleida o Albacete (figura 4e). 
FIGURA 4

ESQUEMATIZACIÓN DE LOS DIFERENTES TIPOS DE AM

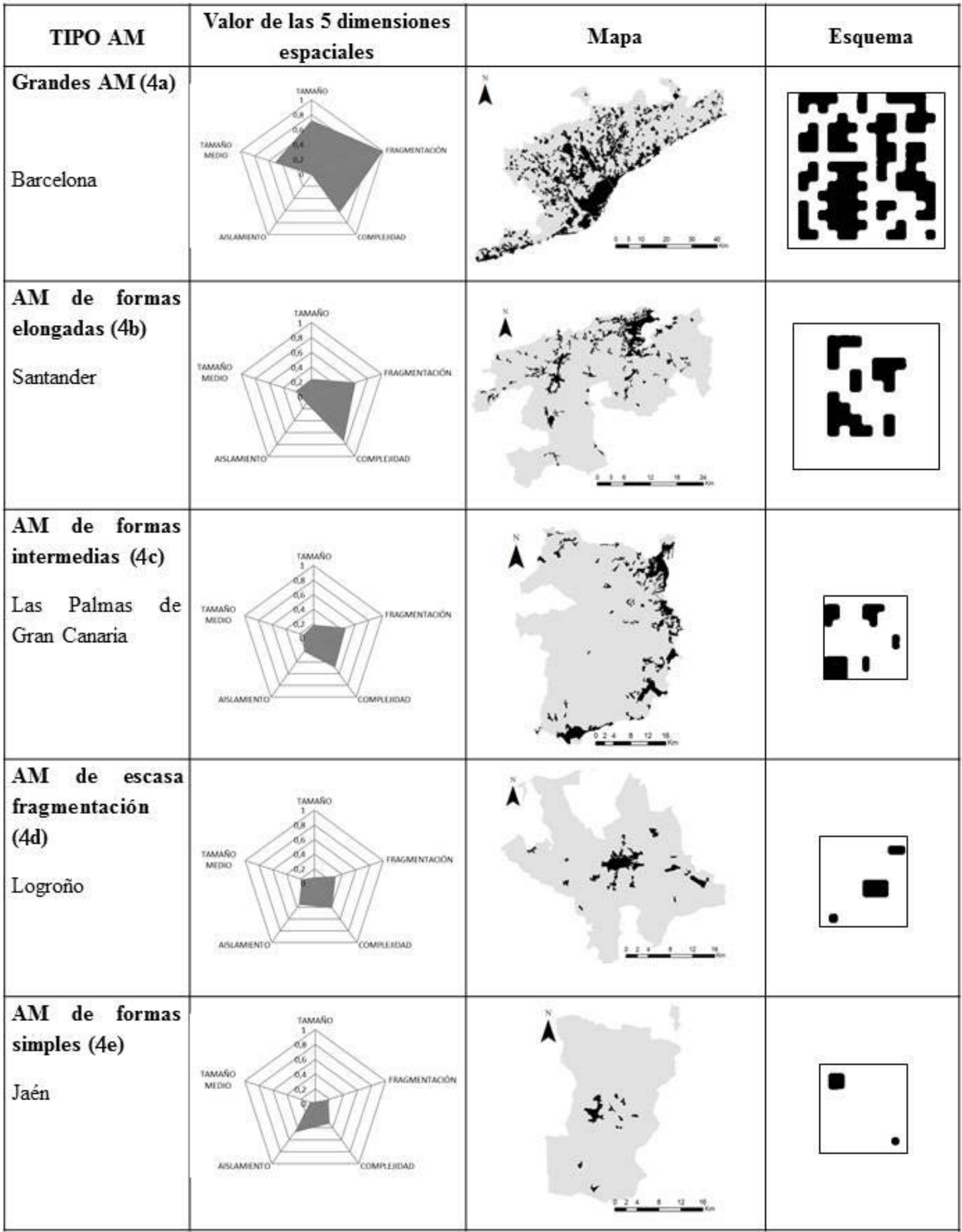




\subsection{Los cambios en la clasificación a lo largo del período} 1990-2000. Procesos de transformación de las AM

Los resultados de las clasificaciones de las AM para el año 1990 y 2000, junto con la anteriormente comentada clasificación para 2006, mostraron tanto la estabilidad de algunas AM, que se mantuvieron en el mismo grupo durante las tres fechas, como los cambios de otras, que pasaron de un grupo a otro. A este respecto, como muestra la figura 5 , se observa una tendencia a la transformación de AM desde los estados más iniciales (AM de formas simples, por ejemplo) hacia los grupos de AM de formas intermedias y de escasa fragmentación, que ven incrementado su número.

De esta forma, algo más de la mitad de las AM que fueron clasificadas en los grupos de "AM de formas simples" o "AM de escasa fragmentación" en 1990, han ido desarrollándose y dando lugar a formas más complejas hasta 2006, donde se han incluido en los grupos de "AM de formas intermedias" $\mathrm{y}$ "AM de formas elongadas". Estas transformaciones, entendidas como cambios de categoría en la clasificación de diferentes fechas, pueden ser interpretadas como procesos de cambio en la estructura del paisaje de las AM. A continuación, se describen algunos de los principales procesos detectados.

\section{Proceso 1. Desarrollo de AM de formas simples}

Este proceso afecta, aproximadamente, a la mitad de las 14 AM pertenecientes al grupo de las "AM de formas simples", que se han transformado lo suficiente para ser clasificadas en varios grupos de AM de mayor complejidad en la forma. Concretamente, durante el periodo 1990-2000, las AM de formas simples de Pamplona, León, Valladolid, Ourense y Manresa han evolucionado pasando a ser clasificadas como "AM en de escasa fragmentación", debido al aumento de la fragmentación (factor 2), complejidad de las formas (factor 3 ) y sobre todo a la disminución drástica de la distancia media entre áreas urbanas (factor 4), como consecuencia de la aparición de nuevas teselas entre los fragmentos urbanos preexistentes (figura 6).

FIGURA 5

CLASIFICACIÓN GENERADA PARA 1990 Y 2000
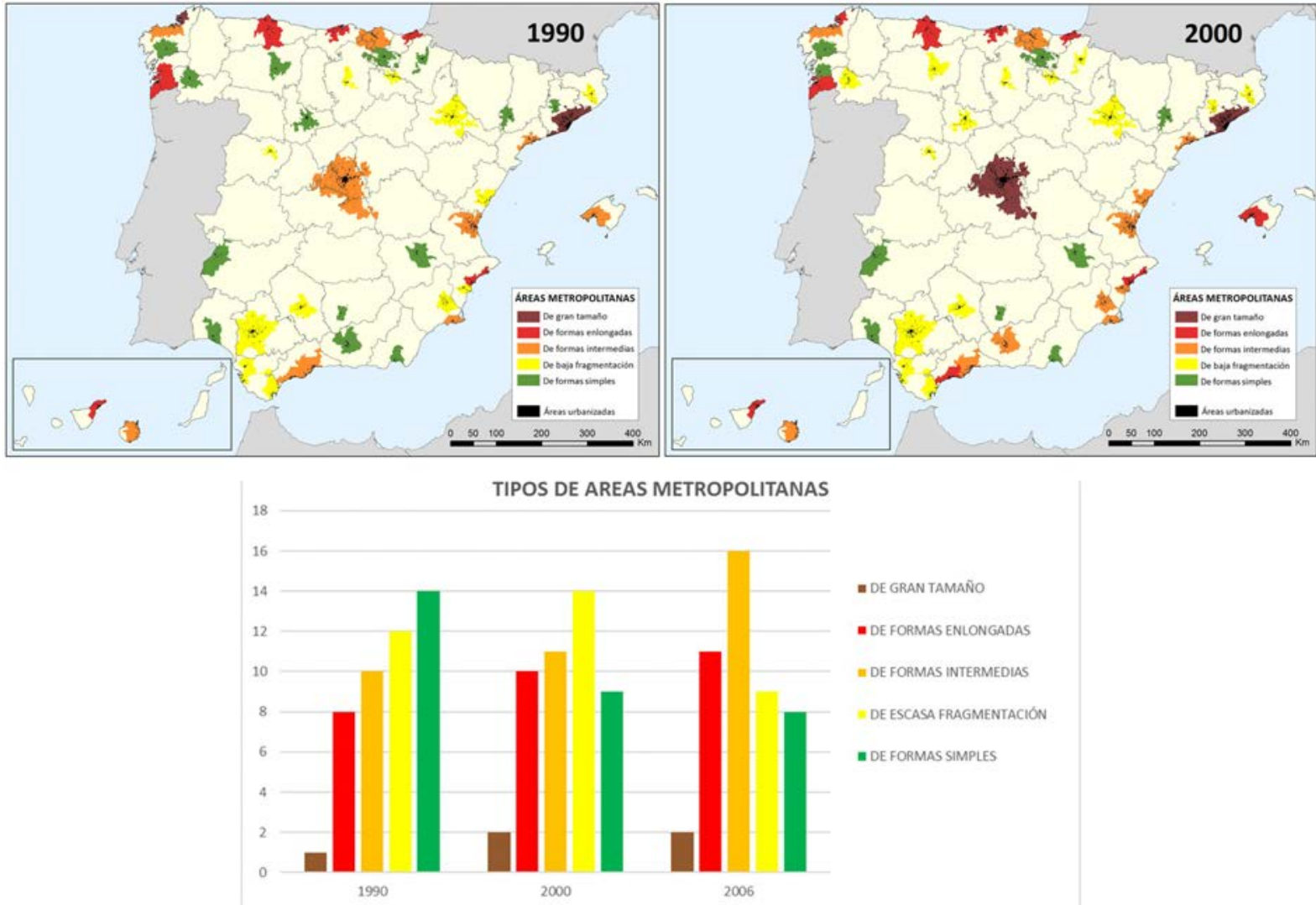
FIGURA 6

FRAGMENTACIÓN Y DISPERSIÓN EN AM DE FORMAS SIMPLES

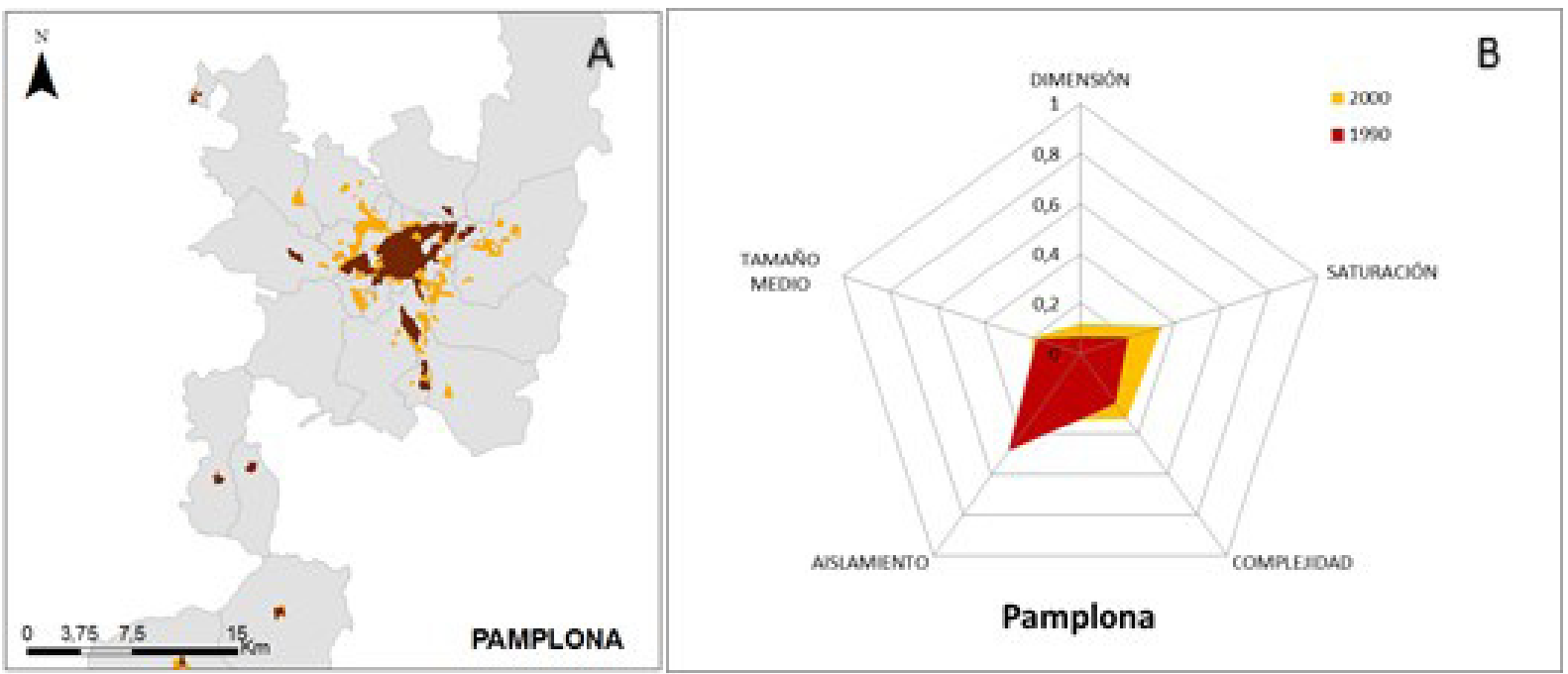

Proceso 2: Aparición de nuevas teselas en AM de escasa fragmentación

Este proceso, parecido al anterior, afecta AM como Murcia, Castellón y Elche, debido al aumento, principalmente, en los factores de fragmentación (factor 2) y tamaño (factor 1 ), así como la reducción del factor de aislamiento de las teselas. Esto se debe fundamentalmente al incremento del número de teselas que forman el área metropolitana, que provoca una menor distancia entre teselas y una mayor fragmentación (figura 7), produciéndose así su evolución hacia "AM de formas intermedias". En algunos casos, como son el de Elche y Murcia, se produce la aparición de las nuevas teselas urbanas también con formas elongadas, dando lugar a continuos lineales (incremento del factor 3). En algunos casos, estas nuevas construcciones Ilegan a conectar zonas preexistentes, anteriormente separadas, por lo que la distancia entre ellas disminuye y se crean núcleos de mayor tamaño medio (factor 5).

FIGURA 7

FRAGMENTACIÓN DE AM DE ESCASA FRAGMENTACIÓN

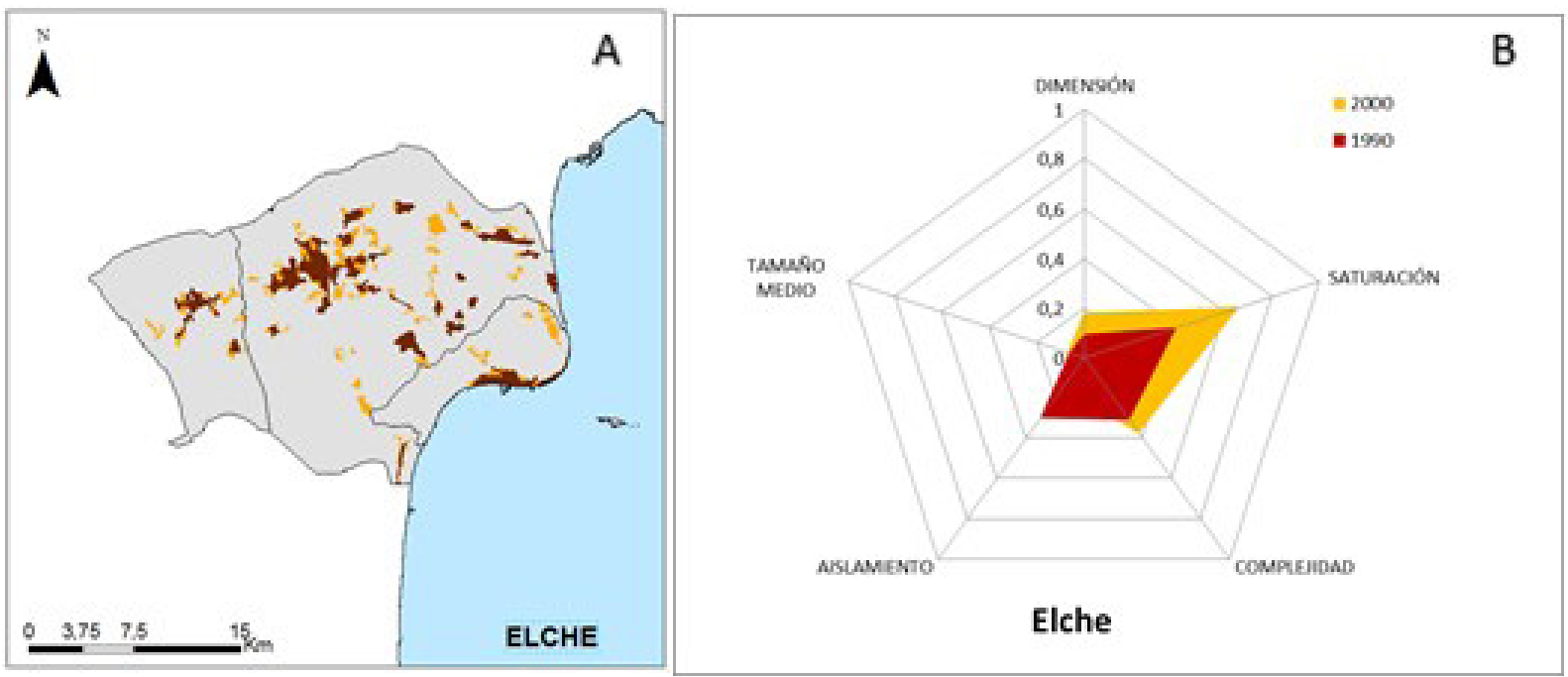


Proceso 3: Conurbación litoral y dispersión en el interior en AM intermedias

Protagonizado por AM de formas intermedias, como Palma de Mallorca y Marbella en el período 1990-2000, que se trasforman en "AM de formas elongadas", en las que se ha producido un crecimiento en torno a la franja costera adyacente a las zonas ya urbanizadas dando lugar a procesos de conurbación que generan fragmentos urbanos de mayor tamaño y formas elongadas (más complejas). Sin embargo, en las zonas del interior destaca la aparición de nuevas teselas urbanas distribuidas por el territorio que incrementan el número de fragmentos urbanos. El resultado de este proceso es, el incremento de los factores de tamaño (factor 1), fragmentación (factor 2), complejidad de las teselas (factor 3 ) y tamaño medio de las mismas (factor 5), así como la disminución del aislamiento de las teselas (factor 4) (figura 8).

\section{Proceso 4. Proceso de agregación y coalescencia}

Este proceso se pone de manifiesto fundamentalmente en el período 2000-2006, afectando a AM pertenecientes a diferentes grupos. Así, lo han experimentado AM de formas simples en 2000 como Almería, que pasa a ser clasificada en 2006 como "AM de escasa fragmentación"; "AM de escasa fragmentación" en 2000 como Zaragoza, Córdoba, Salamanca, Burgos, Pamplona y León, que pasan a ser clasificadas en 2006 como "AM de formas intermedias"; el AM de Málaga, que de ser clasificada en 2000 como "de formas intermedias", es clasificada en 2006 como AM "de formas elongadas"; y finalmente el caso de Granada, que pasa de "AM de formas simples" a ser clasificada en el año 2000 como un
"AM de formas intermedias". Estas transformaciones se deben a un aumento principalmente en el factor de complejidad (factor 3) y tamaño medio (factor 5) (figura 9) como consecuencia de crecimientos agregados a las teselas existentes que dan lugar a conurbaciones en el entorno de elementos lineales, de la línea de costa (Málaga), o a núcleos preexistentes (Granada).

\section{Proceso 5. El caso del AM Madrid}

Además de los cambios de categorías de las AM que tienen como resultado los procesos descritos anteriormente, existe un cambio cuyos resultados merecen ser comentados detenidamente por lo anómalo de su comportamiento. Se trata de la clasificación del AM de Madrid-Alcalá Henares como AM de formas elongadas en el año 1990, en lugar de haber sido asignada al grupo de Grandes AM, tal y como sí que fue clasificada en 2000 y 2006, como sucede con Barcelona para las tres fechas.

A este respecto, es posible señalar que la delimitación del AM de Madrid-Alcalá de Henares, al igual que para el resto de $A M$, se realiza de acuerdo con los criterios de Feria (2008), a partir de datos del censo de 2001. Sin embargo, tal y como se observa en la figura 10, la extensión de dicha delimitación es tan grande para el AM de Madrid-Alcalá de Henares, que las zonas urbanas en 1990 suponían un porcentaje reducido de la extensión de su delimitación, con lo cual la densidad de teselas (PD), muy correlacionada con el factor de fragmentación (factor 2), mostró unos valores más bajos de los esperables, lo que la situó en la clasificación de 1990 en el nivel de AM de formas intermedias.

FIGURA 8.

EJEMPLO DEL PROCESO 3

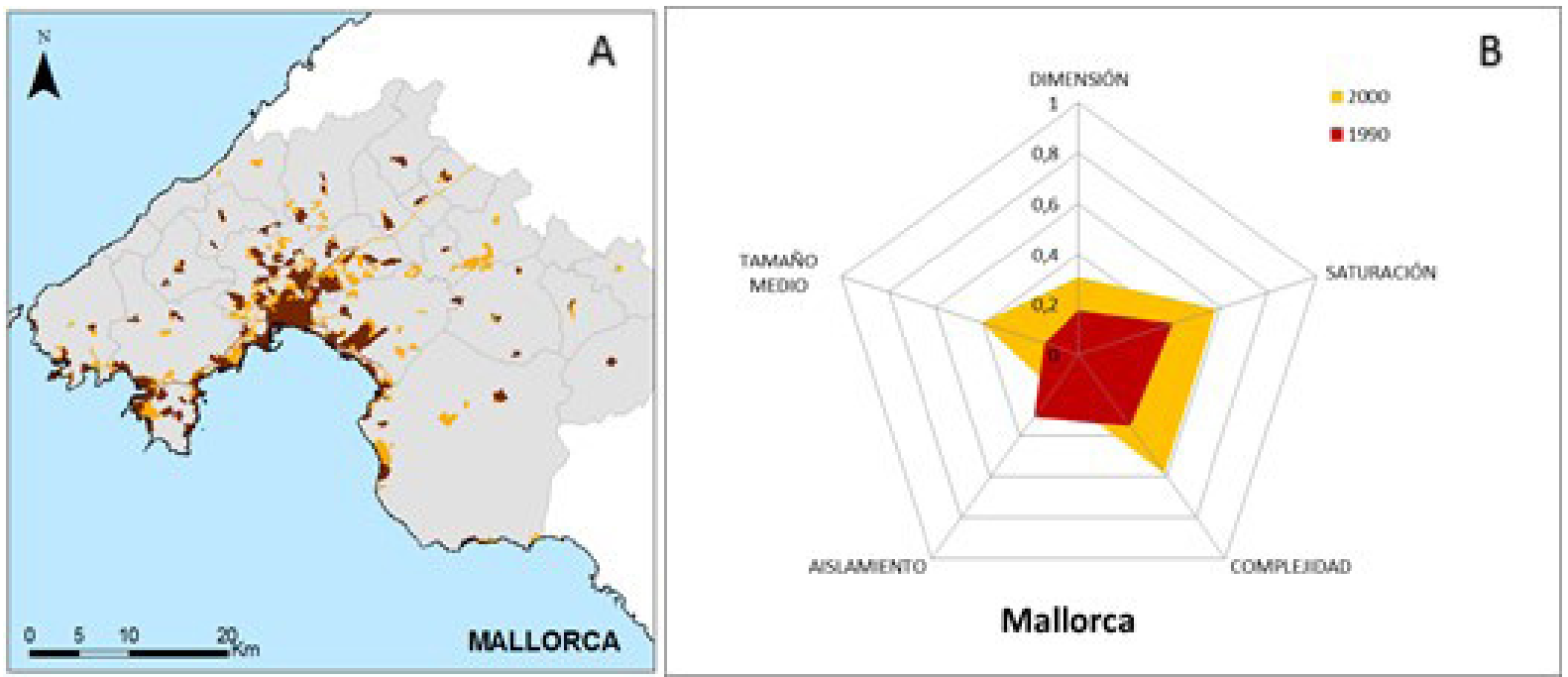


FIGURA 9.

PROCESO DE AGREGACIÓN Y COALESCENCIA

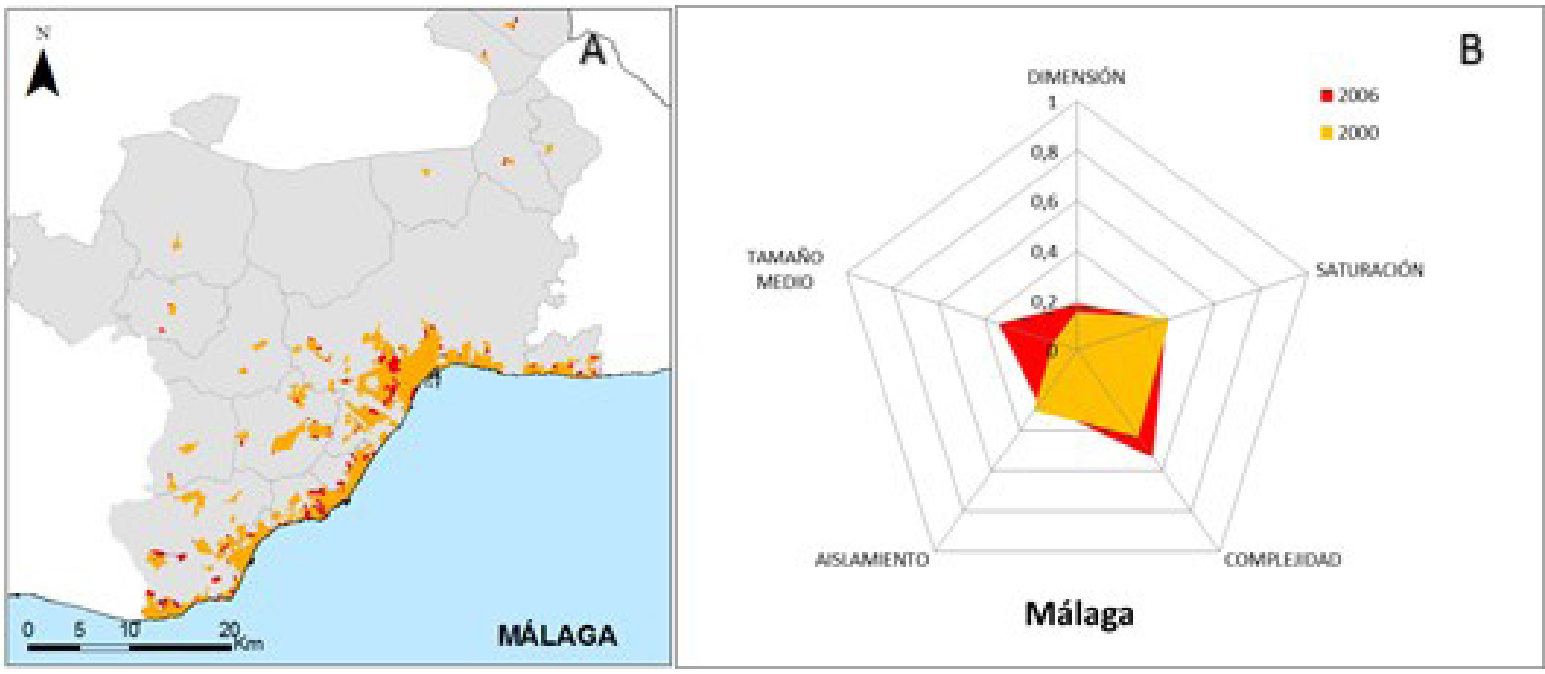

Probablemente este hecho se debe a: i) el extraordinario crecimiento y transformación de Madrid-Alcalá de Henares de 1990 a 2000 (figura 10), que la llevó a modificar incluso la extensión de sus límites en dicho período, como queda de manifiesto en el gran incremento del factor tamaño (factor 1), fragmentación (factor 2), complejidad (factor 3) y tamaño medio (factor 5), acompañado de pérdida de aislamiento (factor 4). Este proceso es similar al proceso de dispersión anteriormente descrito, pero con una dimensión mucho mayor; ii) la delimitación de las AM empleada, basada en movimientos pendulares, que no tiene una relación directa con las formas y patrones de ocupación, lo que podría condicionar en parte los resultados.

Por todo, ello es posible considerar a Madrid-Alcalá de Henares como perteneciente al grupo de Grandes AM en las tres fechas, pero con la concurrencia de un proceso de dispersión de gran escala acontecido en el período de 1990 a 2000 (figura 10).

FIGURA 10.

INCREMENTO DE LA DISPERSIÓN EN MADRID-ALCALÁ DE HENARES

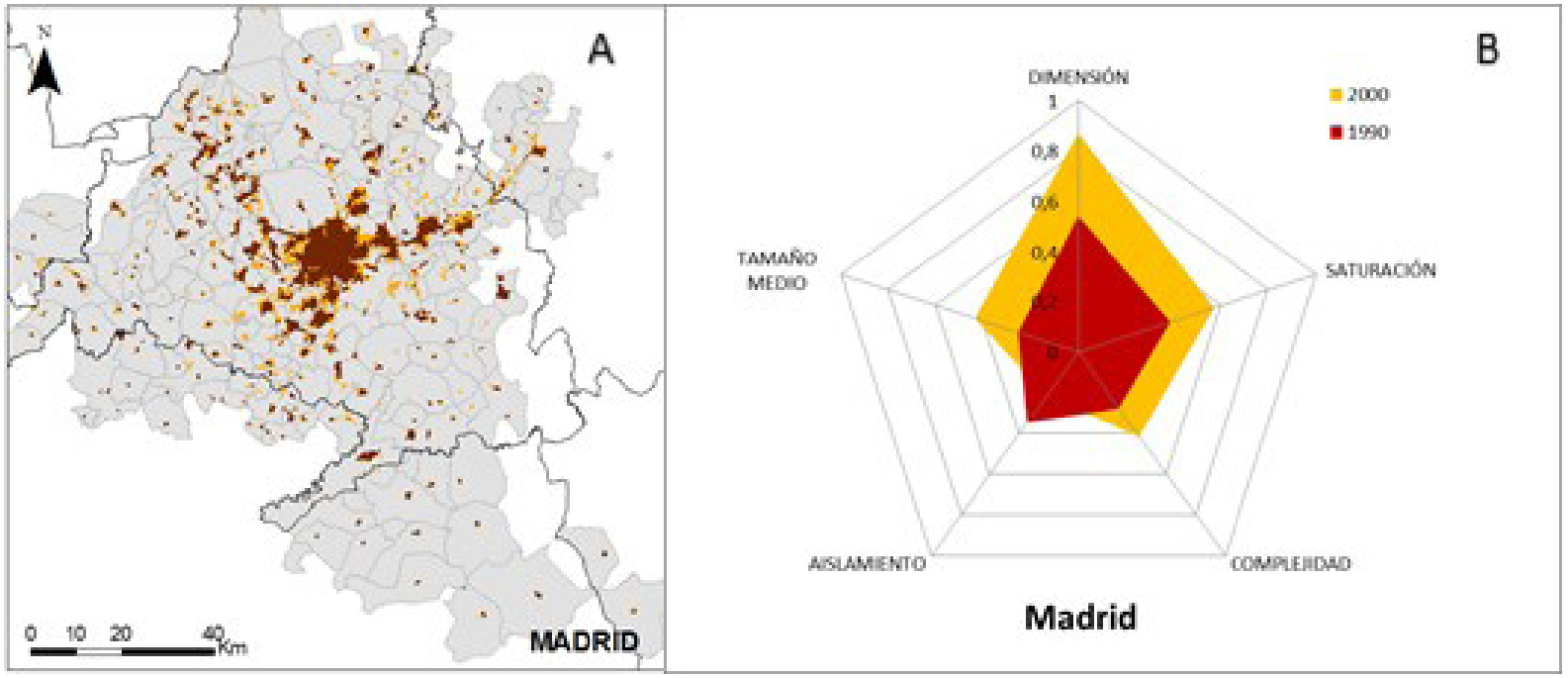




\section{Discusión}

\subsection{Sobre los resultados de la clasificación y los pro- cesos identificados}

El análisis realizado ha permitido identificar aquellas dimensiones espaciales que permiten explicar la variabilidad de la forma urbana de las AM españolas, así como clasificarlas en tres fechas. Posteriormente, la clasificación multitemporal y los cambios de categoría experimentados han permitido inferir procesos de transformación de las AM como consecuencia del crecimiento urbano.

En lo que respecta a las dimensiones identificadas, cinco han sido los factores o dimensiones que han permitido explicar la mayor parte de la variabilidad de las AM españolas. Algunas de ellas, como la densidad de bordes o de teselas, así como el aislamiento o el tamaño medio de las teselas, coinciden con las obtenidas por Cushman et al (2008) para estudios de paisaje en Estados Unidos. Otras, como por ejemplo el tamaño, también han sido seleccionadas como criterios de clasificación de AM en otros trabajos (ver Feria, 2013), por lo que se pueden interpretar como factores relevantes que se repiten a la hora de describir las formas urbanas de espacios metropolitanos.

En relación con la clasificación, se han agrupado las AM en cinco categorías atendiendo a sus características espaciales. Los grupos de AM de gran tamaño, AM de formas simples, o los dominados por formas elongadas, son probablemente los más fácilmente reconocibles. En ellos se incluyen, respectivamente. las grandes AM españolas (Madrid y Barcelona), las AM de desarrollo más incipiente (Almería, Albacete, Jaén, entre otras) o las AM litorales caracterizadas por su crecimiento a lo largo de la línea de costa. No obstante, los otros dos grupos (AM de formas intermedias, y AM de escasa fragmentación), son probablemente grupos heterogéneos de una interpretación menos intuitiva, ya que incluyen a AM de carácter muy diverso. Así, por ejemplo, las AM de Sevilla y Logroño, muy diferentes, se incluyen en el grupo de AM de escasa fragmentación. A este respecto, el tamaño de la delimitación del AM puede haber influido en que aquellas AM que presentaban delimitaciones más extensas hayan tenido valores artificialmente bajos de la dimensión 2 (fragmentación de las teselas).

En lo que respecta a los procesos, se han descrito tanto procesos de dispersión, como procesos de coalescencia o agregación. Los procesos de dispersión afectan generalmente a AM de formas más simples, y por tanto que se encuentran en etapas de desarrollo más incipiente. Dichas AM comienzan a experimentar procesos de suburbanización, que dan lugar a nuevas teselas en el entorno de los núcleos principales. Sufren por tanto un aumento de la superficie urbana, del número de teselas y de la densidad de las mismas, a la vez que disminuye la distancia media a la tesela más próxima, así como generalmente también el tamaño medio de las teselas. Por su parte, los procesos de coalescencia aparecen tanto en las AM de mayor desarrollo, como en las AM fuertemente condicionadas por la topografía o por la proximidad a la línea de costa. En ellas aparecen por tanto fenómenos de conurbación y de relleno intersticial de las teselas preexistentes. Se producen así incrementos del tamaño medio de las teselas y en ocasiones también de la complejidad de las formas.

Estos dos tipos de procesos, bien descritos y caracterizados en Dietzel, Herold, Hemphill y Clarke (2005), en lo que respecta a la evolución de las métricas, se reproducen en la dinámica de expansión de las AM españolas.

\subsection{Sobre algunos errores detectados en la clasifica- ción y "procesos ficticios"}

A pesar del interés de los citados resultados, existen algunos de ellos de carácter anómalo que merecen ser comentados. Así, además del citado caso del AM de Madrid, aparecen dos casos de asignación de $A M$ a un grupo de clasificación que resulta del todo anómalo. Concretamente, el AM de El Ferrol y Pontevedra fueron clasificadas en el año 1990como "AM de gran tamaño" y "AM de formas elongadas" respectivamente, para posteriormente ser clasificadas en el año 2000 como AM "de formas elongadas" y "de formas simples". Ambos cambios de categoría se deben a procesos ficticios producto de problemas en la cartografía CLC y a las propias características de la ocupación urbana en las AM de Galicia. A este respecto, la clasificación de El Ferrol como "AM de gran tamaño" se debe al cartografiado como zonas urbanas de extensas áreas que únicamente incluían algunas teselas de ocupación urbana de baja densidad (ciudad difusa, ve Dalda, García y García, 2006). Estas teselas urbanas ficticias dieron lugar a grandes manchas continuas de uso "urbano" con bordes muy sinuosos, de elevada complejidad de formas y tamaños medios elevados, que presentaban a su vez elevada fragmentación (elevada densidad de teselas), lo que, a pesar de su tamaño muy reducido, la llevaron a ser clasificada junto con Barcelona. 
Este problema se solventó parcialmente al aumentar la precisión de los datos de CLC en años posteriores, que implicaron la eliminación de grandes superficies de la categoría de zonas urbanas. De este modo, lo que en 1990 era erróneamente un gran continuo urbano, se desagregó en áreas de menor tamaño medio, y con menor superficie urbana total, que provocaron que en años sucesivos fuera catalogada como "AM de formas elongadas", categoría mucho más adecuada a sus características. Sin embargo, este error en la CLC 1990 emerge en el presente estudio como un proceso ficticio, que no se corresponde con un cambio real de las formas de las zonas urbanas. Errores similares a estos han sido descritos para la cartografía CLC en algunos otros trabajos como por ejemplo los de Bach et al (2006) o Catalá et al. (2008).

En este mismo sentido, es posible que algunos de los otros procesos identificados y descritos anteriormente se puedan haber producido, al menos parcialmente, como consecuencia de errores en la clasificación de los usos urbanos en CLC en alguna de las fechas analizadas. Igualmente, cierto tipo de procesos de crecimiento urbano que se producen a una escala más detallada tampoco sean identificados, ya que la cartografía CLC no consigue detectarlos. (AguileraBenavente et al, 2014).

Por ello, sería interesante realizar análisis similares empleando cartografías de mayor detalle, como por ejemplo SIOSE, que permitirían identificar procesos de dispersión urbana de pequeño tamaño que no son recogidos por CLC. Igualmente, conviene no obviar el hecho de que los procesos identificados emanan de la inclusión de cada AM en una categoría de las obtenidas en el análisis de conglomerados, y que dicho análisis puede ser sensible, entre otros aspectos, al número de agrupamientos y algoritmo de clasificación seleccionado.

\section{NOTAS}

1. También resulta interesante, a nivel europeo, la metodología DEGURBA para la definición del grado de urbanización y la identificación de gradientes urbano-rurales. Ver https://ec.europa. eu/eurostat/web/gisco/geodata/reference-

\section{Bibliografía}

Aguilera, F. Valenzuela, L.M. y Botequilha-Leito, A. (2011). Landscape metrics in the analysis of urban land use patterns: a case study in a Spanish metropolitan area. Landscape and Urban Planning, 99, 226-238.

\section{CONCLUSIONES}

El presente trabajo constituye un estudio morfométrico pionero en España, que ha permitido identificar las dimensiones espaciales que caracterizan las formas urbanas de las AM españolas, Para ello se ha empleado, en un enfoque novedoso en España, la cartografía de CLC (con las consabidas ventajas y problemas que implica, algunos de ellos ya discutidos en la sección anterior), así como una selección de métricas que permiten cuantificar características espaciales de las teselas urbanas. La metodología empleada permitió además realizar una clasificación multitemporal de las dichas AM en función de sus formas, así como la identificación y caracterización de procesos de crecimiento urbano, que engloban procesos de dispersión urbana, así como de agregación y coalescencia.

No obstante, sería interesante complementar este trabajo desde diversas perspectivas: i) continuar los análisis multitemporales de cambios en la clasificación de las AM mediante la extensión del estudio a fechas más recientes, por ejemplo empleando la nueva cartografía CLC para 2012 y 2018; ii) la exploración del uso de otras fuentes cartográficas, como por ejemplo la cartografía SIOSE, que igualmente aporta una dimensión multitemporal pero con una mayor resolución temática y espacial; iii) incorporar análisis de otros aspectos relacionados y/o explicativos de las formas urbanas, tales como aspectos socioeconómicos de diversa índole (Reis, Silva y Pinho, 2016), relaciones de movilidad, distribución espacial de las diferentes funciones y usos urbanos etc., que permitieran circunscribir los resultados no solo a la descripción y cuantificación de las formas de la "mancha" urbana.

\section{Agradecimientos}

Este trabajo ha contado con la financiación del proyecto SIMURBAN 2 (CO2012-38158-C02-01), financiado por el Ministerio de Economía y Competitividad.

data/population-distribution-demography/degurba

2. En el texto se emplean indistintamente el término dimensión y factor.

Aguilera-Benavente, F., Botequilha-Leitão A y Díaz, E. (2014). Detecting multi-scale urban growth patterns and processes in the Algarve region (Southern Portugal). Applied Geography, 53, 234-245. 
Aguilera-Benavente, F. y Botequilha-Leitão, A. (2012). Selección de métricas de ecología del paisaje mediante ACP para la caracterización de los procesos de alteración del paisaje del Algarve (Portugal). Geofocus. Revista Internacional de Ciencia y Tecnología de la Información geográfica, 12, 93-121.

Antrop, M. (2004). Landscape change and the urbanization process in Europe. Landscape and Urban Planning, 67, 9-26.

Bach, M., Breuer, L., Frede, H. G., Huisman, J. A., Otte, A. y Waldhardt, R. (2006). Accuracy and congruency of three different digital land-use maps. Landscape and urban planning, 78 (4), 289-299.

Boix-Domenech, R. (2007). “Concepto y delimitación de AM: una aplicación a las AM de España". Seminario Las grandes AM españolas en una perspectiva comparada, Sevilla. http://urban.uab.es/ references/2007/07002.pdf (Fecha de consulta: 18/4/2017).

Botequilha Leitao, A. y Ahern, J (2002). Applying landscape ecological concepts and metrics in sustainable landscape planning. Landscape and Urban Planning, 59, 65-93.

Burriel, E.L. (2013). Subversion of land-use plans and the housing bubble in Spain. Urban Research \& Practice, 4(3), 232-249.

Carreras, J.M.; Otero, M. y Ruiz, E. (2009). Aglomeraciones Metropolitanas Europeas. Papers 50, Barcelona, Institut d'Estudis Regionals i Metropolitans de Barcelona (IERMB), 250 pp.

Catalá Mateo, R., Bosque Sendra, J., y Plata Rocha, W. (2008). Análisis de posibles errores en la base de datos CORINE Land Cover (1990-2000) en la Comunidad de Madrid. Estudios Geográficos, 69, 81-104.

CE (2016). The State of European Cities 2016. Luxemburgo, Oficina de Publicaciones de la Unión Europea, http://ec.europa.eu/cities-report (Fecha de consulta: 18/4/2017).

Chen, Y., Li, X., Zheng, Y., Guan, Y., y Liu, X. (2011). Estimating the relationship between urban forms and energy consumption: A case study in the Pearl River Delta, 2005-2008. Landscape and Urban Planning, 102, 33-42.

Cushman, S.A., McGarigal, K. y Neel, M.C. (2008). Parsimony in landscape metrics: Strength, universality and consistency. Ecological Indicators, 8, 691703.
Dalda Gonzalo, J.L.; García Docampo, M. y García Harguindey, J. (2006). Cidade difusa en Galicia. Dirección Xeral de Urbanismo, Santiago de Compostela, $126 \mathrm{pp}$.

Deng, J. S., Wang, K., Hong, Y., y Qi, J. G. (2009). Spatio-temporal dynamics and evolution of land use change and landscape pattern in response to rapid urbanization. Landscape and Urban Planning, 92, 187-198.

Dietzel, C., Herold, M., Hemphill, J.J. y Clarke, K.C. (2005). Spatio-temporal dynamics in California's Central Valley: Empirical links to urban theory. International Journal of Geographical Information Science, 19 (2), 175-195.

EEA (2006). Land accounts for Europe 1990-2000. EEA Report №11/2006. Office for Official Publications of the European Communities, Luxemburgo. https://www.eea.europa.eu/publications/eea_report_2006_11 (Fecha de consulta: 18/4/2017).

Feria Toribio, J.M. (2004). Problemas de definición de las AM en España. Boletín de la Asociación de Geógrafos Españoles, 38, 85-100.

Feria Toribio, J.M. (2008). Un ensayo metodológico de definición de las AM en España a partir de la variable residencia-trabajo. Investigaciones Geográficas, 46, 49-68.

Feria Toribio, J. M. (2013). Hacia una taxonomía de las AM Españolas. Boletín de la Asociación de Geógrafos Españoles, 63, 499-506.

Feria Toribio, J. M. (2015). Los modelos de organización y dinámicas espaciales metropolitanas en Andalucía. Cuadernos Geográficos, 54 (2), 196-219.

Grimm, N.B., Faeth, S.H., Golubiewski, N.E., Redman, C.L., Wu, J., Bai, X., y Briggs, J.M. (2008). Global change and the ecology of cities. Science, 319, 756-760.

Herold, M., Couclelis, H., y Clarke K.C. (2005). The role of spatial metrics in the analysis and modelling of urban land use change. Computers, Environment and Urban Systems, 29, 369-399.

Huang, J., Lu, X.X., y Sellers, J. M. (2007). A global comparative analysis of urban form: Applying spatial metrics and remote sensing. Landscape and Urban Planning, 82, 184-197.

McGarigal, K., Cushman, S.A. y Ene, E. (2012). FRAGSTATS v4: Spatial Pattern Analysis Program for Categorical and Continuous Maps. Computer 
software program produced by the authors at the University of Massachusetts, Amherst. http:// www.umass.edu/landeco/research/fragstats/ fragstats.html (Fecha de consulta: 18/4/2017).

Ministerio de Fomento (2016). Atlas Digital de las Áreas Urbanas de España 2016. https://www.fomento.gob.es/portal-del-suelo-y-politicas-urbanas/atlas-estadistico-de-las-areas-urbanas/atlasdigital (Fecha de consulta: 18/4/2017).

Miramontes, A. y Vieira, T.M. (2016). Las Áreas urbanas en la Península Ibérica. Un ejercicio de delimitación. Papeles de Geografía, 62, 47-62.

Pascual, V.; Aguilera, F. y Salado, M.J. (2014). "Incidencia de la resolución espacial y temática en la aplicación de métricas espaciales a la descripción morfológica de las AM españolas". Actas del XVI Congreso Nacional de Tecnologías de la Información Geográfica. Alicante.

Pham, H. M., y Yamaguchi, Y. (2011). Urban growth and change analysis using remote sensing and spatial metrics from 1975 to 2003 for Hanoi, Vietnam. International Journal of Remote Sensing, 32(7), 1901-1915.

Reis, J.P., Silva, E.A. y Pinho, P. (2016). Spatial metrics to study urban patterns in growing and shrinking cities. Urban Geography, 37, 246-271.

Roca Cladera, J., Moix Bergadá, M y Arellano Ramos, B. (2012). El sistema urbano en España. Scripta Nova. Revista electrónica de geografía y ciencias sociales, 396.

Salom, J. y Albertos J.M. (2010). “Densidad de la red viaria y forma urbana: Delimitación del espacio urbano en ocho Aglomeraciones españolas". En Feria Toribio, J.M y Albertos Puebla, J.M. (coords.) La ciudad metropolitana en España: procesos urbanos en los inicios del siglo XXI. Madrid: Civitas, $442 \mathrm{pp}$.

Schwarz, N. (2010). Urban form revisited-Selecting indicators for characterising European cities. Landscape and Urban Planning, 96, 29-47. 